\title{
Rôle des oiseaux sauvages dans la transmission et la dispersion des virus de l'influenza aviaire : apport de l'éco-épidémiologie dans les écosystèmes afro-tropicaux
}

\author{
Nicolas Gaidet et Alexandre Caron* \\ CIRAD UPR AGIRs département ES TA C-22/E, Campus international de Baillarguet 34398 Montpellier Cedex 5 France
}

\begin{abstract}
Résumé - La diffusion intercontinentale du virus de l'influenza aviaire (VIA) hautement pathogène H5N1 en 2005-2006 a déclenché un effort de recherche important sur l'épidémiologie des VIA chez les oiseaux sauvages et à l'interface entre oiseaux sauvages et domestiques. En effet, les oiseaux sauvages hébergent les souches de VIA faiblement pathogènes qui une fois transmises à la volaille peuvent évoluer vers des souches de plus forte pathogénicité qui constituent un risque économique et de santé publique. Les travaux réalisés au cours des dix dernières années en Afrique ont permis d'étudier les VIA dans des contextes écologiques et épidémiologiques peu explorés. Les résultats indiquent que les VIA persistent dans les écosystèmes afrotropicaux tout au long de l'année et sont probablement maintenus dans les communautés d'oiseaux sauvages. L'écologie de la transmission de ces VIA dans les régions afro-tropicales présente cependant des particularités. Ainsi, le rôle des limicoles ne semble pas aussi crucial dans la maintenance des VIA que dans les écosystèmes nord-américains. La maintenance des VIA n'est peut-être pas uniquement dépendante des canards sauvages et une grande diversité d'espèces de la communauté d'oiseaux sauvages semble participer à la circulation de ces virus. Finalement, certaines espèces peuvent jouer le rôle de relais, créant le lien épidémiologique entre canards sauvages (l'hôte de maintenance) et les populations de volaille domestique (l'hôte cible). Ces travaux ont donc permis de produire les premières esquisses des modes de circulation et de persistance des VIA dans les communautés d'oiseaux sauvages et domestiques en Afrique, mais ont aussi contribué à stimuler la recherche globale sur les VIA en proposant des hypothèses originales et des méthodes pour les tester.
\end{abstract}

Mots clés : influenza aviaire / oiseaux sauvages / interface sauvage/domestique / Afrique / écologie de la santé

\begin{abstract}
The role of wild birds in the transmission and spread of avian influenza viruses: eco-epidemiological approaches in afro-tropical ecosystems. The intercontinental spread of highly pathogenic avian influenza viruses (AIV) in 2005-2006 triggered an important research effort on the epidemiology of AIV in wild birds and at the wild/domestic bird interface. Wild birds are the host of the majority of low pathogenic AIV that may evolve into highly pathogenic strains once introduced into poultry populations, causing a risk for animal and public health. The work undertaken during the last 10 years in afro-tropical ecosystems explored the eco-epidemiology of AIV in wild and domestic birds. AIV do persist across the year in Africa, and are probably maintained in wild bird communities, but the patterns of species and seasonal variations differ from what has been described on other continents. The role of shorebirds does not seem to be as crucial in the maintenance of AIV when compared to some North American ecosystems. AIV maintenance appears to be depending not only on wild ducks but also on a wide diversity of host species in the wild bird community. Finally, some species could act as bridge hosts, creating an epidemiological link between wild ducks (maintenance host) and domestic poultry populations (susceptible host). These works provide an updated understanding of the transmission dynamics of AIV in African wild and domestic birds and contributed to the global research on AIV by raising new hypotheses and unfolding new methods to test them.
\end{abstract}

Keywords: avian influenza / wild birds / wild/domestic interface / Africa / disease ecology

\footnotetext{
* Auteur de correspondance : Alexandre.caron@cirad.fr
} 


\section{Introduction}

L'hiver 2005-2006 a vu l'apparition de foyers d'infection de grippe aviaire dans la volaille et les oiseaux sauvages causés par le virus H5N1 hautement pathogène (HP) en Asie, en Europe puis en Afrique. Ce virus circulait en Extrême-Orient depuis déjà plusieurs années. L'arrivée de ce virus dans de nombreuses populations de volaille à travers le monde a fait l'objet d'une résonance médiatique forte par son impact sur les filières avicoles domestiques mais aussi par l'inquiétude d'un risque pandémique qu'il a réactivé peu après l'épidémie du SARS (2003).

Si aujourd'hui ces virus n'ont plus la faveur des médias, ils restent néanmoins des pathogènes majeurs pour les populations de volaille et constituent toujours des agents potentiellement zoonotiques. L'année 2015 a ainsi enregistré le plus grand nombre de cas d'infection chez l'homme dans le monde (143 personnes infectées dont 43 décès) par le virus H5N1 HP depuis 2003, principalement en Égypte et en Asie du Sud-Est. Ce virus a été également à l'origine de nombreux foyers en 2015 dans la volaille et les oiseaux sauvages dans de nombreux pays d'Asie, du Moyen-Orient, d'Afrique et d'Europe de l'Est. L'Amérique du Nord n'a pas été non plus épargnée avec de très nombreux foyers de virus HP (H5N1, H5N2, H5N6 et H5N8). Enfin, au cours de l'hiver 2015-2016, la France a connu une flambée de foyers d'infection dans des élevages de volaille du Sud-Ouest (81 foyers détectés à ce jour depuis novembre 2015 dans dix départements), associés à différentes souches de virus HP (H5N1, H5N2 et H5N9). Que sait-on aujourd'hui du rôle des oiseaux sauvages dans la perpétuation des virus de l'influenza aviaire (VIA) et du risque de transmission à la volaille ?

\subsection{Les virus de l'influenza aviaire}

Les VIA sont des orthomyxovirus à ARN, classés en soustypes selon les deux glycoprotéines de surface de la particule virale, l'hémagglutinine (HA) et la neuraminidase (NA), respectivement impliquées dans la fixation et la libération du virus de la cellule hôte. Au total, 16 HA (H1-H16) et neuf NA (N1-N9) ont été caractérisées chez les oiseaux sauvages. Les mécanismes de dérive antigénique (mutations lors de la réplication) et de réassortiment (échange de segments de génome entre souches) sont à l'origine de la forte variabilité génétique des VIA. Les VIA sont également classés en virus faiblement pathogène (FP) et HP selon leur pathogénicité chez le poulet et leurs caractéristiques moléculaires. La transmission se fait par contact direct entre hôtes ou indirect via l'environnement.

Il est communément admis que seuls les VIA FP circulent chez les oiseaux sauvages, à quelques rares exceptions près (Gaidet et al., 2008) chez qui ils ne provoquent généralement pas de signes cliniques (Olsen et al., 2006 ; Kuiken, 2013). Les VIA HP, responsables d'une forte mortalité chez la volaille, n'ont pas d'hôte de maintenance connu chez les oiseaux sauvages. Ces VIA HP sont généralement considérés avoir pour origine des VIA FP circulant chez les oiseaux sauvages qui, après introduction et adaptation à la volaille domestique, acquièrent par mutation et recombinaison des caractéristiques génétiques leur conférant une haute pathogénicité (Munster et al., 2010 ; Beato et Capua, 2011).

\section{2 Écologie de la transmission des VIA chez les oiseaux sauvages}

Dès 2005 , la question du rôle des oiseaux sauvages dans la dispersion des VIA HP s'est posée en Europe et en Afrique où les oiseaux migrateurs eurasiatiques passent l'hiver. Quelles espèces sont concernées ? Quel est le rôle des communautés d'oiseaux sauvages dans la maintenance des VIA FP ? Quel est le risque que les oiseaux migrateurs dispersent des virus d'un continent à l'autre ? Finalement, quelles sont les espèces impliquées dans la transmission des VIA vers les oiseaux domestiques?

La quasi-totalité des informations disponibles en 2005 sur ces questions concernaient les zones tempérées et boréales de l'hémisphère nord où des populations d'oiseaux sauvages avaient été suivies (Fouchier et al., 2003 ; Krauss et al., 2004 ; Olsen et al., 2006) et où se situent les laboratoires de référence. Ces études faisaient alors office de matrice principale pour émettre des hypothèses sur les mécanismes épidémiologiques dans le reste du monde. Dans les régions tropicales africaines, en l'absence d'information épidémiologique, il a fallu mettre en place un cadre de recherche combinant les sciences de l'épidémiologie et de l'écologie (Caron et al., 2009). Ces travaux ont donc porté sur l'étude des processus écologiques qui sous-tendent les dynamiques de transmission des pathogènes au sein des populations ou des communautés d'hôtes (Plowright et al., 2008 ; Carver et al., 2009). Cette approche s'est structurée afin de comprendre :

- quels sont les mécanismes de perpétuation des VIA FP chez les hôtes de maintenance que constituent les oiseaux sauvages ;

- quelle est la capacité de dispersion des VIA par les espèces migratrices ;

- quels sont les processus de transmission de ces virus à l'interface entre les oiseaux sauvages et la volaille.

\section{Perpétuation des VIA chez les oiseaux sauvages des zones afro-tropicales}

Les patrons de variations d'infection VIA observés en Europe et en Amérique du Nord ne peuvent pas être directement transposés aux zones tropicales, qui diffèrent en termes d'écologie des hôtes, de saisonnalité et de conditions environnementales (Gaidet, 2015). L'hypothèse est que les mécanismes par lesquels l'écologie des hôtes influence la transmission des VIA peuvent être associés à des facteurs écologiques distincts et produire des dynamiques d'infection distinctes dans les zones tropicales. Dans les zones afrotropicales, les saisons sont déterminées par les variations des précipitations plutôt que des températures, qui sont généralement hautes et relativement constantes tout au long de l'année ; en revanche, la plupart des zones humides afro-tropicales sont soumises à de très fortes variations de leur surface en eau au cours de l'année en raison de précipitations saisonnières et d'un fort taux d'évaporation. De même, la composition des communautés d'oiseaux sauvages, leurs dynamiques de reproduction et de migration diffèrent entre ces zones biogéographiques (Mundava et al., 2012). Enfin, les communautés virales peuvent également être différentes, et l'existence de souches virales spécifiques à ces zones ne peut être écartée. 


\subsection{Détection de la circulation des VIA dans les communautés d'oiseaux sauvages}

Une série de campagnes de terrain a été réalisée entre 2006 et 2010 dans différents grands sites de concentration d'oiseaux d'eau à travers le continent africain (Fig. 1) pour détecter la présence de VIA ( $>15000$ oiseaux capturés et échantillonnés dans 19 pays). La détection de VIA chez de nombreuses espèces et groupes taxonomiques (anatidés - canards -, limicoles, laridés - goélands, sternes -, poules d'eau, passereaux) dans plusieurs grandes zones humides, a révélé que les conditions environnementales sont bien favorables à la circulation des VIA dans les différentes régions du continent africain (Gaidet et al., 2007a, 2007b) contrairement à l'a priori que les températures moyennes élevées en Afrique empêchaient la persistance des VIA. L'isolement de plusieurs sous-types distincts de VIA au sein d'une même population de canards migrateurs a aussi révélé la co-circulation de différents virus, comme dans de nombreux sites européens ou nord-américains, créant les conditions pour une recombinaison entre virus provenant potentiellement de différentes aires géographiques. D'autre part, des VIA ont été détectés chez des canards migrateurs durant les derniers mois de leur période d'hivernage en Afrique de l'Ouest : ce résultat indique que les canards migrateurs hivernants au Sud du Sahara peuvent constituer une source d'introduction de VIA vers l'Europe lors de leur migration au printemps. De même, la détection de VIA chez des oiseaux afro-tropicaux qui restent sur le continent toute l'année suggère un possible maintien de ces virus dans les écosystèmes africains. Enfin, des VIA ont pu également être détectés chez des limicoles paléarctiques pendant l'hivernage et la migration en Afrique, ce qui contraste avec l'absence de VIA rapportée jusqu'alors dans toutes les études sur les limicoles en Europe (Fouchier et al., 2003).

Une série de nouvelles questions ont été posées suite à ces premiers résultats : les virus persistent-ils toute l'année dans les communautés d'oiseaux sauvages des écosystèmes de zones humides afro-tropicales ? Comment expliquer les variations de taux d'infection VIA entre les sites, les saisons et les espèces hôtes ? Quel est le rôle des limicoles, qui constituent la grande majorité des oiseaux d'eau migrateurs reliant l'Eurasie et l'Afrique, dans la dispersion intercontinentale des VIA ?

\subsection{Suivis longitudinaux de la circulation virale dans des communautés d'oiseaux en Afrique}

Des suivis longitudinaux ont été réalisés afin de tester l'existence d'une circulation des VIA tout au long de l'année en zone afro-tropicale dans trois écosystèmes distincts : le banc d'Arguin en Mauritanie, le delta intérieur du Niger au Mali, et le complexe des lacs Manyame et Chivero au Zimbabwe. Les suivis réalisés sur ces trois sites ont permis de mettre en évidence l'existence d'une circulation des VIA FP tout au long de l'année au sein des communautés d'oiseaux sauvages dans des contextes écologiques distincts (zones humides aussi bien côtières que continentales), en particulier durant les saisons où les migrateurs eurasiatiques sont absents (Cappelle et al., 2011 ; Caron et al., 2011). Une circulation lors de différentes saisons a également été détectée dans le site de Mauritanie où les canards sont absents toute l'année, suggérant la contribution d'autres groupes d'hôtes sauvages à la persistance des VIA (Gaidet et al., 2012a). Globalement, dans ces écosystèmes africains, la circulation des VIA existe à un niveau faible, avec des niveaux plus élevés chez les anatidés et pendant les saisons où les migrateurs eurasiatiques sont présents (octobre à mars) mais qui coïncident avec les périodes de forte concentration d'oiseaux. Ces résultats indiquent qu'une persistance annuelle des VIA est possible dans les communautés d'hôtes des régions afro-tropicales et suggèrent l'existence d'un cycle endémique des VIA en Afrique.
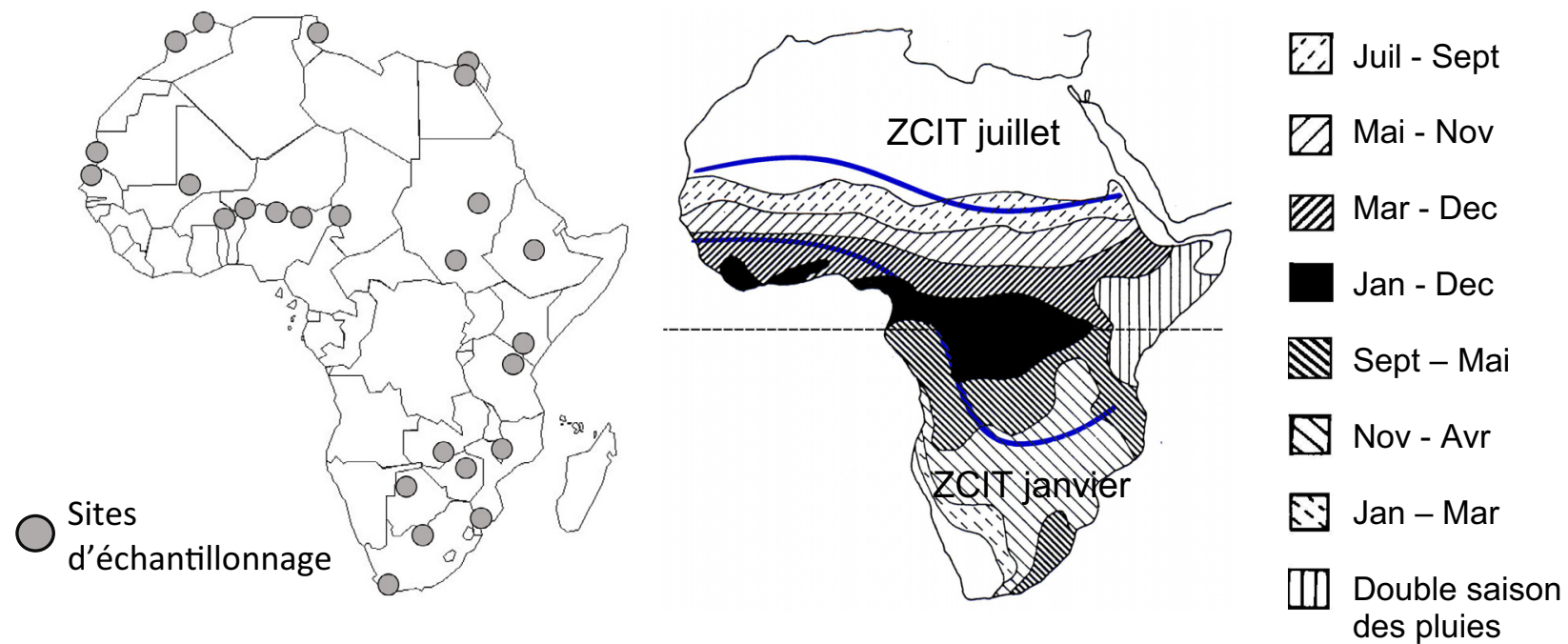

Fig. 1. Distribution continentale des sites d'échantillonnage et des saisons des pluies en relation avec la position saisonnière de la zone de convergence intertropicale (ZCIT).

Fig. 1. Continental distribution for sampling sites and timing of the wet season and seasonal position of the Inter-Tropical Convergence Zone (ITCZ).

Figure adaptée de Jones (1995). 


\subsection{Déterminants écologiques influençant la transmission des VIA au sein des communautés de canards sauvages}

L'analyse des données collectées à l'échelle continentale sur environ 8400 anatidés sauvages dans 16 sites afrotropicaux révèle que les variations de taux d'infection VIA sont associées à plusieurs facteurs écologiques, soit à l'échelle de l'hôte (le groupe taxonomique), soit à l'échelle de la communauté (densité locale d'anatidés, saison d'hivernage des anatidés migrateurs) (Gaidet et al., 2012b).

Le groupe taxonomique apparaît être un meilleur prédicteur des variations d'infection entre espèces que les facteurs écologiques d'exposition au virus: un taux d'infection significativement plus élevé a été trouvé chez les espèces de canards du genre Anas que chez les autres espèces d'anatidés, quel que soit leur comportement alimentaire ou migrateur. Jusque-là, les études avaient systématiquement rapporté un taux d'infection plus important chez les canards de surface que chez les canards plongeurs ou brouteurs, selon l'hypothèse que ces deux derniers sont moins exposés au virus dans l'environnement (Olsen et al., 2006 ; Munster et al., 2007). La détection d'un taux d'infection VIA supérieur chez les espèces du genre Anas par rapport aux autres canards de surface, suggère que des facteurs intrinsèques peuvent contribuer, au-delà des facteurs écologiques d'exposition, à la probabilité d'infection d'une espèce au sein d'une communauté d'hôtes.

Les taux d'infection VIA ont été trouvés positivement corrélés à la densité locale des anatidés plutôt qu'à leur abondance (nombre d'individus). Les différentes variables climatiques testées (température, évapotranspiration, aridité) se sont révélées être de faibles prédicteurs des taux d'infection. Ces résultats suggèrent une prédominance de la transmission directe (une transmission interindividuelle densité-dépendante) par rapport à la transmission indirecte (via des virus persistant dans un réservoir environnemental) en zone afro-tropicale, à l'opposé du modèle proposé pour les zones tempérées où la transmission environnementale semble avoir une plus forte contribution que la transmission interindividuelle (Rohani et al., 2009).

En zones boréale et tempérée, le regroupement prémigratoire en fin d'été des canards migrateurs, dont une forte proportion de juvéniles immunologiquement naïfs (Munster et al., 2007), est le facteur écologique majeur proposé pour expliquer le pic saisonnier de prévalence de VIA observé chaque automne chez les canards sauvages, via l'augmentation $\mathrm{du}$ taux de contact et la transmission interindividuelle favorisées par cette agrégation sociale. En zones afrotropicales, cette agrégation saisonnière résulte d'un autre processus, lors de l'assèchement des zones humides au cours de la saison sèche lorsque les anatidés se regroupent sur les quelques zones humides permanentes. Toutefois, les variations saisonnières des taux d'infection dans les écosystèmes afrotropicaux n'ont pu être associées avec la période d'agrégation des oiseaux en fin de la saison sèche. Cela pourrait s'expliquer par le fait que le regroupement saisonnier des oiseaux se fait de manière plus graduelle en zone tropicale (suite à la réduction progressive des surfaces en eau disponibles pendant la saison sèche) que dans les zones tempérées et boréales (rassemblement social pré-migratoire relativement bref) ; de la même manière, le recrutement de jeunes oiseaux immunologiquement naïfs se fait de manière plus progressive en zones afrotropicales (en raison de l'étalement et de la non-synchronisation de la période de reproduction des différentes espèces d'anatidés africains) à la différence des zones tempérées et boréales (Mundava et al., 2016). La dynamique progressive de ces deux facteurs pourrait expliquer l'existence d'une circulation VIA à faible niveau et moins variable entre saisons, mais continue tout au long de l'année dans les écosystèmes afro-tropicaux (Gaidet, 2015).

Si le rôle des canards africains comme hôte de maintenance des VIA est indiscutable, cela ne veut pas forcément dire que le rôle du reste de la communauté d'oiseaux sauvages n'a pas d'influence non seulement sur l'écologie des VIA mais aussi en particulier sur leur maintenance. La comparaison des profils de circulation des VIA au sein de différentes communautés d'oiseaux sauvages dans des écosystèmes d'Afrique australe a permis de montrer que d'autres groupes d'oiseaux définis par leur rôle dans l'écologie des VIA (ex., maintenance, introduction/dispersion) peuvent aussi contribuer au maintien des VIA dans certains écosystèmes. Parmi ces groupes classés en fonction de leur capacité à introduire et à maintenir les VIA, on trouve par exemple les laro-limicoles afro-tropicaux et le groupe des oiseaux résidants a priori non impliqués dans la fonction de maintenance (rest of community - ROC dans l'article -, c'est-à-dire ni les canards ni les laro-limicoles) (Caron et al., 2012). Coupler l'étude des communautés d'oiseaux sauvages (composition, abondance relative) avec les études épidémiologiques est indispensable pour aborder la complexité des dynamiques épidémiologiques dans les systèmes multi-hôtes et en particulier les VIA dans les communautés ornithologiques qui peuvent regrouper plusieurs centaines d'espèces.

\subsection{Déterminants écologiques influençant la transmission des VIA chez les laro-limicoles}

Les laro-limicoles (goélands, sternes et petits échassiers) sont généralement considérés comme le deuxième groupe d'hôtes des VIA le plus important, après celui des anatidés (Arnal et al., 2015). Globalement et localement, le taux d'infection chez les laro-limicoles est toutefois faible (environ $1 \%$ ), à l'exception notable d'un foyer récurrent de circulation à forte prévalence $(>10 \%)$ chez les tourne-pierres à collier (Arenaria interpres) pendant leur halte migratoire en mai dans la baie du Delaware (États-Unis) (Krauss et al., 2010). Cette combinaison unique espèce-site-saison constitue le seul hot spot de circulation de VIA chez des limicoles répertorié au monde. La baie du Delaware est l'un des plus grands sites de concentration de laro-limicoles au monde (environ 1,5 million d'oiseaux s'y rassemblent chaque année lors des haltes migratoires).

En Afrique, aucun hot spot de circulation de VIA chez les laro-limicoles n'a été détecté, malgré la vaste étendue géographique et taxonomique des oiseaux échantillonnés (69 espèces de laro-limicoles appartenant à dix familles distinctes échantillonnées dans 25 pays), en particulier dans les grands sites de concentration de laro-limicoles sur les sites d'hivernage ou de migration (delta du Nil-Égypte, banc d'Arguin-Mauritanie, delta du Sénégal, delta du Niger-Mali, 
plaines de la Kafue-Zambie) (Gaidet et al., 2012a). De plus, les variations de taux d'infection entre sites et saisons n'ont pu être associées avec l'abondance locale des laro-limicoles. Cette absence de hot spot d'infection est particulièrement notable au banc d'Arguin, et en particulier dans sa population de tournepierres à collier. Ce site partage en effet plusieurs caractéristiques avec la baie du Delaware : également côtier, il accueille plus de deux millions de laro-limicoles en hivernage, présents à forte densité sur les zones d'alimentation, dont la plus grande abondance de tourne-pierres à collier de l'ancien monde. La forte concentration d'oiseaux ne semble donc pas être le facteur écologique déterminant, ou en tout cas une condition suffisante, à la présence d'un hot spot d'infection. Ces résultats indiquent également qu'une forte prévalence n'est pas systématiquement détectée chez les tourne-pierres à collier.

La présence de VIA a néanmoins été détectée chez des larolimicoles à différentes saisons dans plusieurs sites de zone côtière: cette circulation faible mais continue dans un écosystème qui présente de fortes contraintes pour la survie $\mathrm{du}$ virus dans l'environnement (forte salinité et dessiccation par l'exposition au vent et aux radiations solaires en l'absence de végétation, et submersion régulière de la zone intertidale d'alimentation) suggère là aussi une prédominance de la transmission directe entre oiseaux par rapport à la transmission environnementale au sein de la communauté d'hôtes. Ces résultats confortent aussi l'idée que le rôle d'une espèce ou d'un groupe d'espèces dans l'épidémiologie des VIA peut varier d'un écosystème à l'autre et que les caractéristiques intrinsèques de l'hôte (ex., réceptivité à l'infection ou capacité à répliquer le virus) n'expliquent pas à elles seules son rôle dans l'épidémiologie des VIA.

\section{Capacité de dispersion des VIA HP par les canards migrateurs}

La mobilité d'un hôte pendant la période d'infectiosité et d'excrétion par un virus, mais aussi sa sensibilité à l'infection vont déterminer sa capacité à disperser ce pathogène. La plupart des espèces d'anatidés sont des espèces migratrices qui parcourent de très longues distances (plusieurs milliers de kilomètres) entre leurs zones de reproduction et leurs zones d'hivernage. L'infection par les VIA FP est généralement considérée comme asymptomatique, les oiseaux infectés ne montrant pas de signes cliniques. La proximité phylogénétique entre souches FP isolées dans différentes régions du globe chez des oiseaux sauvages (ex., Dugan et al., 2008 ; Koehler et al., 2008) suggère qu'il existe une dispersion intercontinentale des VIA FP par les oiseaux migrateurs. De même, des études montrent le lien qui existe entre les routes migratoires des oiseaux et la structuration phylogéographique des souches de VIA isolées chez les oiseaux sauvages (Lam et al., 2012).

À la suite de la dispersion rapide, en 2005 et 2006, des VIA H5N1 HP depuis l'Est de l'Asie jusqu'à l'Europe et l'Afrique, et la découverte à la même époque de foyers d'infection $\mathrm{H} 5 \mathrm{~N} 1$ chez des anatidés migrateurs avec une forte mortalité (Liu et al., 2005), ces oiseaux ont été désignés comme principaux responsables de la dispersion du virus. S'il est vrai que ces anatidés sont capables de déplacements rapides sur de longues distances et constituent les principaux hôtes des VIA FP, leur capacité à disperser ce virus constitue un sujet controversé.

\subsection{Suivi des déplacements d'un oiseau naturellement infecté par un VIA hautement pathogène}

Lors d'une campagne de prélèvement au Nord du Nigéria (plaine d'inondation d'Hadejia-Nguru), des virus H5N2 avec un génotype HP ont été détectés chez plusieurs oiseaux ne présentant pas de signes cliniques (Gaidet et al., 2008). Ces oiseaux appartenaient à deux espèces communes d'anatidés africains (un dendrocygne veuf, Dendrocygna viduata, et six oies de Gambie, Plectropterus gambensis), qui ne migrent pas en dehors du continent. Parmi ces oiseaux, un dendrocygne veuf s'est trouvé être l'un des oiseaux équipés d'une balise satellite pour une étude menée en parallèle sur la mobilité des canards africains.

Dans les jours suivant la détection de son infection, cet oiseau a effectué des déplacements relativement courts (1 à $7 \mathrm{~km}$ par jour) mais similaires à ceux effectués par l'autre dendrocygne suivi lors du même protocole, et comparables aux patrons de déplacements connus chez cette espèce (Petrie et Rogers, 1997). Le suivi des déplacements de cet oiseau a révélé qu'il avait survécu à l'infection, fournissant une rare évidence d'une infection naturelle non létale par un VIA HP chez un oiseau sauvage. Cet oiseau a réalisé plusieurs grands déplacements (de 140 à $175 \mathrm{~km}$ par jour) dans les semaines suivantes, mais au-delà de la période de persistance de l'excrétion de VIA (généralement $<7$ jours chez les anatidés), ce qui n'a pas permis d'évaluer sa capacité de dispersion du virus sur de longues distances.

\section{2 Évaluation indirecte du potentiel de dispersion par les anatidés migrateurs}

La télémétrie par satellite est une méthode de plus en plus utilisée pour suivre les déplacements d'animaux sauvages dans de vastes régions isolées. Les données de localisation qui sont fournies tous les deux à quatre jours permettent l'analyse des mouvements individuels avec une haute résolution temporelle compatible avec la durée de l'infection virale. Un ensemble de canards, oies et cygnes migrateurs (228 oiseaux de 19 espèces) ont été équipés de balises satellites dans les principales régions où les VIA H5N1 HP ont été détectés (Afrique, Asie et au Moyen-Orient). Leurs déplacements ont été analysés afin d'évaluer la distance maximum et la fréquence auxquelles un anatidé migrateur pouvait disperser un VIA HP (Gaidet et al., 2010).

Cette analyse repose sur une approche indirecte : elle consiste à mesurer la portée et la fréquence des déplacements des oiseaux suivis par satellite en fonction de la durée d'infection asymptomatique. Cette durée d'infection asymptomatique mesurée en condition expérimentale (le nombre de jours pendant lesquels l'oiseau excrète du virus sans signe clinique) est en moyenne de quatre jours. La distance maximum entre toutes les localisations enregistrées pendant une fenêtre de temps de quatre jours a été calculée pour chaque oiseau, de manière itérative tout au long de la période de suivis par satellite, pour évaluer le potentiel de dispersion des oiseaux.

Les résultats indiquent que, chez toutes les espèces d'anatidés migrateurs étudiées, un oiseau a bien la capacité de disperser le virus sur de longues distances (Gaidet et al., 2010) : en moyenne, ces oiseaux sont capables, selon les 
espèces, de parcourir entre 500 et $1500 \mathrm{~km}$ en seulement quatre jours. Toutefois, l'analyse montre que la probabilité qu'un tel événement se produise reste très faible : la période d'infection d'un anatidé migrateur doit pour cela coïncider avec la période où l'oiseau s'engage dans un grand déplacement, événement qui ne se produit en moyenne, selon les espèces, que de cinq à 15 jours par an pour un déplacement de $500 \mathrm{~km}$ ou plus. En outre, lorsque l'on allonge la durée potentielle des fenêtres de temps d'infection, l'analyse révèle que la distance maximum couverte n'augmente presque plus au-delà de cinq jours. Ce résultat révèle que la migration chez les anatidés est constituée de longs vols rapides et directs, mais peu nombreux, et entrecoupés de haltes migratoires plus longues que la durée de l'infection chez ces oiseaux. Cela signifie qu'il est très peu probable que ces oiseaux puissent disperser du virus lors de plusieurs longs vols migratoires consécutifs. Pendant la migration, les escales des oiseaux sur leurs sites de repos sont généralement plus longues que la période d'infection et d'excrétion virale ; aussi, les oiseaux ne peuvent pas répandre le virus sur de longues distances en un seul vol. La dispersion du virus à l'échelle continentale suppose donc une transmission relais de l'infection entre plusieurs oiseaux migrateurs successivement infectés. Le rassemblement d'anatidés migrateurs en grand nombre sur certains sites le long des couloirs de migration, oiseaux d'origine et de destinations distinctes, avec des tempos d'arrivée et de départ non synchronisés, peut faciliter cette transmission en relais.

\section{Processus de transmission des VIA entre les oiseaux sauvages et domestiques}

\subsection{Du besoin de l'étude de l'interface sauvage/ domestique}

Si la diversité des VIA FP se trouve chez les oiseaux sauvages et que ces VIA FP peuvent évoluer en HP une fois introduits dans des élevages de volaille domestique, la compréhension des processus qui opèrent à l'interface entre oiseaux sauvages et domestiques constitue un élément crucial. Cette interface est importante lorsque des élevages de type extensif fournissent des espaces de contacts directs ou via l'environnement entre oiseaux sauvages et domestiques, en particulier autour de sources d'eau ou d'aliments. C'est le cas des élevages extensifs d'autruche en Afrique australe (principalement Afrique du Sud et Zimbabwe) où des souches de VIA, parentes des souches de VIA HP chez les autruches, ont été retrouvées chez des canards sauvages capturés dans les élevages (Abolnik, 2007). Ce risque de transmission a aussi été caractérisé dans le delta intérieur du Niger à l'aide d'un suivi satellite de canards sauvages s'approchant de points d'eau à proximité des villages (Cappelle et al., 2011). Dans le cas de bâtiments d'élevage intensif de volaille, un faible niveau de biosécurité peut permettre des contacts directs ou indirects (dans le temps) entre oiseaux sauvages et domestiques. Caractériser ces contacts et les espèces sauvages impliquées nécessite des protocoles adaptés et jusqu'ici peu développés. Ces protocoles combinent les observations d'oiseaux sauvages en périphérie des bâtiments d'élevage et dans les zones humides proches, ainsi que des suivis épidémiologiques.

\subsection{Le concept d'hôte relais, reliant les hôtes de maintenance et les hôtes cibles}

Dans de nombreux cas, il n'existe pas de contacts directs entre les hôtes de maintenance des VIA (tels que les canards sauvages) et les oiseaux domestiques (hôtes cibles), les canards sauvages restant dans les zones humides loin des élevages. La transmission est-elle possible quand deux hôtes ne sont pas ou rarement en contact?

La transmission d'un pathogène entre deux hôtes qui ne sont pas en contact et ne partagent pas le même environnement peut se produire par l'intermédiaire d'un hôte relais (bridge host) si ce dernier est successivement en contact avec les deux autres hôtes. Si l'existence d'hôtes relais a depuis longtemps été proposée, la caractérisation de leur fonction et leur identification au sein des communautés d'hôtes n'a été que rarement étudiée jusqu'à présent. Un cadre conceptuel a été développé pour définir la fonction épidémiologique des populations relais (Caron et al., 2015). Un hôte relais doit présenter différentes propriétés :

- avoir des caractéristiques écologiques l'amenant à être exposé au pathogène ;

- être en contact (direct ou indirect) avec les hôtes de la communauté de maintenance et les hôtes cibles ;

- être compétent pour le pathogène (réceptif à l'infection et capable de d'excréter le pathogène en quantité suffisante pour infecter un autre hôte);

- être incapable de maintenir seul le pathogène dans un écosystème (sinon cette espèce serait qualifiée d'hôte de maintenance) (Haydon et al., 2002).

Cette fonction relais permet de créer un lien potentiellement infectieux entre des hôtes qui ne sont pas en contact : ce lien peut être spatial (entre des hôtes présents dans des habitats ou zones géographiques distincts), temporel (entre des hôtes présents dans un même écosystème, ou naïfs à l'infection, à des saisons distinctes), ou comportemental (entre des hôtes sans contact direct dans le cas d'un pathogène à transmission uniquement directe).

\section{3 Évaluation du rôle des espèces relais dans la transmission de pathogènes entre populations d'hôtes}

Une étude de terrain a été réalisée pour identifier les espèces relais potentiel des VIA au Zimbabwe, dans un écosystème où cohabitent une large variété d'oiseaux sauvages et différents systèmes d'élevage, intensifs et extensifs (Caron et al., 2010, 2014). La réalisation de comptages d'oiseaux sur des sites épidémiologiques clés (zones humides, périphérie de villages, élevages intensifs) a permis d'identifier des espèces d'oiseaux capables de créer un lien infectieux entre populations sauvages et domestiques et de caractériser les saisons les plus à risque de transmission de VIA. Ces espèces d'oiseaux potentiellement relais appartiennent pour la plupart à des familles généralement considérées comme des hôtes sauvages accidentels des VIA (passereaux, hérons) (Olsen et al., 2006).

Dans un deuxième temps, un échantillonnage ciblé de ces espèces potentiellement relais a permis de détecter une infection VIA dans deux des trois espèces (l'hirondelle rustique Hirunda rustica, et le travailleur à bec rouge Quelea quelea) 
présentant le plus fort taux de contact relais lors des comptages. Cette approche de détermination des hôtes relais semble donc efficace pour identifier et mesurer le risque de transmission des VIA entre oiseaux sauvages et domestiques. Il est à noter qu'un tout petit nombre d'espèces représente la grande majorité de ce potentiel de transmission relais. Cela suggère que des stratégies de gestion (ex., limitation des contacts) ciblées sur un petit nombre d'espèces sauvages pourraient permettre de limiter significativement le risque de transmission vers les populations domestiques.

\section{Conclusion}

De nombreux aspects de la connaissance de l'écologie de la transmission des VIA chez les oiseaux sauvages, admis par la communauté scientifique avant l'avènement du H5N1 HP en 2005 , ont évolué ou ont été remis en question au cours des dix dernières années. L'évolution rapide des connaissances sur l'écologie des VIA suggère qu'il est nécessaire d'être prudent quant à la généralisation des processus écologiques qui influencent la transmission des VIA dans l'avifaune. Les résultats des études menées en zones afro-tropicales indiquent ainsi que les mécanismes par lesquels l'écologie des hôtes influence la transmission d'un pathogène peuvent être associés à des déterminants écologiques distincts selon le contexte écologique et ainsi produire des patrons de dynamique d'infection différents. Ces résultats suggèrent également qu'il est nécessaire de prendre en compte la communauté des hôtes dans son ensemble, au-delà des espèces considérées comme les principaux hôtes de maintenance, ainsi que leurs interactions écologiques au sein d'un écosystème, afin d'explorer les processus de transmission de pathogènes multi-hôtes tels que les VIA.

L'émergence du virus influenza A(H7N9) en 2013 en Chine a lui aussi apporté son lot de nouveautés : ce virus constitue le premier cas de VIA zoonotique présentant des caractéristiques moléculaires d'un virus FP. Â ce jour, 736 cas humains ont été confirmés et ont provoqué la mort de 283 personnes en Chine. L'émergence du H7N9 FP, comme dans le cas du H5N1 HP, a pour origine une recombinaison de différents virus dont des VIA FP initialement présents chez les oiseaux sauvages (Lam et al., 2013). Cependant, les oiseaux sauvages ou domestiques trouvés infectés par ce virus H7N9 FP ne présentent aucun signe clinique (Zhao et al., 2014) et, ainsi, ne peuvent jouer le rôle de sentinelle comme dans le cas des VIA HP, ce qui rend le contrôle et la prévention de ce virus particulièrement délicat.

Le fait que de nouveaux VIA zoonotiques ne cessent d'émerger à partir d'un pool génétique de virus circulant, entre autres, chez les oiseaux sauvages, puis qu'ils soient dispersés et deviennent endémiques dans certaines régions du monde, souligne la nécessité de maintenir un effort de surveillance ciblé et coordonné au niveau mondial des VIA circulant dans l'avifaune (Machalaba et al., 2015). De plus, le rôle des interactions entre oiseaux sauvages et domestiques dans l'émergence de nouvelles souches VIA HP souligne l'importance d'améliorer notre compréhension des processus de transmission et d'évolution des VIA à l'interface entre oiseaux sauvages et volaille. Toutefois, si les précurseurs de souches VIA HP sont à chercher chez les oiseaux sauvages, il est généralement admis que ces souches n'acquièrent leur caractéristique de haute pathogénicité qu'après avoir été introduites dans les populations d'oiseaux domestiques au sein desquelles elles évoluent par mutation en souche VIA HP (Munster et al., 2010 ; Beato et Capua, 2011). Le fait que l'émergence de souches VIA HP se produise au sein de la volaille doit donc aussi amener à s'interroger sur les pratiques d'élevage qui favorisent ce processus : les conditions d'élevage intensif, dans lesquelles des oiseaux génétiquement très proches ont été sélectionnés pour un rythme de croissance élevé et sont maintenus à de très fortes densités (jusqu'à 22 poulets $/ \mathrm{m}^{2}$ ) qui augmentent la fréquence des contacts entre individus, peuvent favoriser la sélection et l'émergence des souches les plus pathogènes parmi les virus provenant des oiseaux sauvages.

Remerciements. Ces travaux ont été réalisés dans le cadre d'expertise et de projets financés par la FAO et le ministère des Affaires étrangères français (FSP GRIPAVI).

\section{Références}

Abolnik C. 2007. Molecular characterization of H5N2 avian influenza viruses isolated from South African ostriches in 2006. Avian Dis 51: 873-879.

Arnal A, Vittecoq M, Pearce-Duvet J, Gauthier-Clerc M, Boulinier T, Jourdain E. 2015. Laridae: a neglected reservoir that could play a major role in avian influenza virus epidemiological dynamics. Crit Rev Microbiol 41: 508-519.

Beato MS, Capua I. 2011. Transboundary spread of highly pathogenic avian influenza through poultry commodities and wild birds: a review. Rev Sci Tech Off Int Epizoot 30: 51-61.

Cappelle J, Gaidet N, Iverson SA, Takekawa JY, Newman SH, Fofana $\mathrm{B}$, et al. 2011. Characterizing the interface between wild ducks and poultry to evaluate the potential of transmission of avian pathogens. Int J Health Geogr 10: 60.

Caron A, Gaidet N, de Garine-Wichatitsky M, Morand S, Cameron EZ. 2009. Evolutionary biology, community ecology and avian influenza research. Infect Genet Evol 9: 298-303.

Caron A, de Garine-Wichatitsky M, Gaidet N, Chiweshe N, Cumming GS. 2010. Estimating dynamic risk factors for pathogen transmission using community-level bird census data at the wildlife/ domestic interface. Ecol Soc 15: 25.

Caron A, Abolnik C, Mundava J, Gaidet N, Burger CE, Mochotlhoane $\mathrm{B}$, et al. 2011. Persistence of low pathogenic avian influenza virus in waterfowl in a Southern African ecosystem. Ecohealth 8: 109-115

Caron A, de Garine-Wichatitksy M, Ndlovu M, Cumming GS. 2012. Linking avian communities and avian influenza ecology in southern Africa using epidemiological functional groups. Vet Res 43: 73.

Caron A, Grosbois V, Etter E, de Garine-Wichatitsky M. 2014. Bridge hosts for avian influenza viruses at the wildlife/domestic interface: an eco-epidemiological framework implemented in southern Africa. Prev Vet Med 117: 590-600.

Caron A, Cappelle J, Cumming GS, de Garine-Wichatitsky M, Gaidet N. 2015. Bridge hosts, a missing link for disease ecology in multihost systems. Vet Res 46: 83 .

Carver S, Bestall A, Jardine A, Ostfeld RS. 2009. Influence of hosts on the ecology of arboviral transmission: potential mechanisms influencing dengue, Murray Valley encephalitis, and Ross River virus in Australia. Vector Borne Zoonotic Dis 9: 51-64.

Dugan VG, Chen R, Spiro DJ, Sengamalay N, Zaborsky J, Ghedin E, et al. 2008. The evolutionary genetics and emergence of avian influenza viruses in wild birds. PLoS Pathog 4: e1000076. 
Fouchier RA, Olsen B, Bestebroer TM, Herfst S, van der Kemp L, Rimmelzwaan GF, et al. 2003. Influenza A virus surveillance in wild birds in Northern Europe in 1999 and 2000. Avian Dis 47: 857-860.

Gaidet N. 2015. Ecology of avian influenza virus in wild birds in tropical Africa. Avian Dis 60(1s): 296-301.

Gaidet N, Dodman T, Caron A, Balança G, Desvaux S, Goutard F, et al. 2007a. Influenza A viruses in waterbirds in Africa. Emerging Infectious Diseases 13: 626-629.

Gaidet N, Dodman T, Caron A, Balança G, Desvaux S, Goutard F, et al. 2007b. Influenza surveillance in wild birds in Eastern Europe, the Middle East, and Africa: preliminary results from an ongoing FAO-led survey. $J$ Wildl Dis 43: S22-S28.

Gaidet N, Cattoli G, Hammoumi S, Newman SH, Hagemeijer W, Takekawa JY, et al. 2008. Evidence of infection by H5N2 highly pathogenic avian influenza viruses in healthy wild waterfowl. PLoS Pathog 4: e1000127.

Gaidet N, Cappelle J, Takekawa JY, Prosser DJ, Iverson SA, Douglas DC, et al. 2010. Potential spread of highly pathogenic avian influenza $\mathrm{H} 5 \mathrm{~N} 1$ by wildfowl: dispersal ranges and rates determined from large-scale satellite telemetry. J Appl Ecol 47: 1147-1157.

Gaidet N, Ould El Mamy AB, Cappelle J, Caron A, Cumming GS, Grosbois V, et al. 2012a. Investigating avian influenza infection hotspots in old-world shorebirds. PLOS ONE 7: e46049.

Gaidet N, Caron A, Cappelle J, Cumming GS, Balanca G, Hammoumi $\mathrm{S}$, et al. 2012b. Understanding the ecological drivers of avian influenza virus infection in wildfowl: a continental scale study across Africa. Proc R Soc B 279: 1131-1141.

Haydon DT, Cleaveland S, Taylor LH, Laurenson MK. 2002. Identifying reservoirs of infection: a conceptual and practical challenge. Emerg Infect Dis 8: 1468-1473.

Jones PJ. 1995. Migration strategies of Palearctic passerines in Africa. Isr J Zool 41: 393-406.

Koehler AV, Pearce JM, Flint PL, Franson JC, Ip HS. 2008. Genetic evidence of intercontinental movement of avian influenza in a migratory bird: the northern pintail (Anas acuta). Mol Ecol 17: 4754-4762.

Krauss S, Walker D, Pryor SP, Niles L, Chenghong L, Hinshaw VS, et al. 2004. Influenza A viruses of migrating wild aquatic birds in North America. Vector Borne Zoonotic Dis 4: 177-189.

Krauss S, Stallknecht DE, Negovetich NJ, Niles LJ, Webby RJ, Webster RG. 2010. Coincident ruddy turnstone migration and horseshoe crab spawning creates an ecological "hot spot" for influenza viruses. Proc R Soc Lond Ser B 277: 3373-3379.

Kuiken T. 2013. Is low pathogenic avian influenza virus virulent for wild waterbirds? Proc R Soc B Biol Sci 280: 20130990.
Lam TTY, Ip HS, Ghedin E, Wentworth DE, Halpin RA, Stockwell TB, et al. 2012. Migratory flyway and geographical distance are barriers to the gene flow of influenza virus among North American birds. Ecol Lett 15: 24-33.

Lam TT-Y, Wang J, Shen Y, Zhou B, Duan L, Cheung C-L, et al. 2013. The genesis and source of the H7N9 influenza viruses causing human infections in China. Nature 502: 241-244.

Liu J, Xiao H, Lei F, Zhu Q, Qin K, Zhang XW, et al. 2005. Highly pathogenic $\mathrm{H} 5 \mathrm{~N} 1$ influenza virus infection in migratory birds. Science 309: 1206.

Machalaba CC, Elwood SE, Forcella S, Smith KM, Hamilton K, Jebara KB, et al. 2015. Global avian influenza surveillance in wild birds: a strategy to capture viral diversity. Emerg Infect Dis 21: e1-e7.

Mundava J, Caron A, Gaidet N, Couto F, Couto T, de GarineWichatitksy M, et al. 2012. Factors influencing long-term and seasonal waterbird abundance and composition at two adjacent lakes in Zimbabwe. Ostrich 83: 69-77.

Mundava J, Caron A, de Garine-Wichatitsky M, Abolnik C, Mundy P, Gaidet N. 2016. Role of breeding phenology and aggregation of waterfowl on avian influenza dynamics in southern Africa. Ibis 158: $762-775$.

Munster VJ, Baas C, Lexmond P, Waldenstrom J, Wallensten A, Fransson T, et al. 2007. Spatial, temporal, and species variation in prevalence of influenza A viruses in wild migratory birds. PLoS Pathog 3: e61.

Munster VJ, Schrauwen EJ, de Wit E, van den Brand JM, Bestebroer TM, Herfst S, et al. 2010. Insertion of a multibasic cleavage motif into the hemagglutinin of a low-pathogenic avian influenza H6N1 virus induces a highly pathogenic phenotype. $J$ Virol 84: 7953-7960.

Olsen B, Munster VJ, Wallensten A, Waldenstrom J, Osterhaus AD, Fouchier RA. 2006. Global patterns of influenza a virus in wild birds. Science 312: 384-388.

Petrie SA, Rogers KH. 1997. Satellite tracking of white-faced whistling ducks in a semiarid region of South Africa. J Wildl Manag 61: 1208-1213.

Plowright RK, Sokolow SH, Gorman ME, Daszak P, Foley JE. 2008. Causal inference in disease ecology: investigating ecological drivers of disease emergence. Front Ecol Environ 6: 420-429.

Rohani P, Breban R, Stallknecht DE, Drake JM. 2009. Environmental transmission of low pathogenicity avian influenza viruses and its implications for pathogen invasion. Proc Natl Acad Sci USA 106: 10365-10369.

Zhao B, Zhang X, Zhu W, Teng Z, Yu X, Gao Y, et al. 2014. Novel avian influenza $A(H 7 N 9)$ virus in tree sparrow, Shanghai, China, 2013. Emerg Infect Dis 20: 850-853.

Citation de l'article : Gaidet N, Caron A. 2016. Rôle des oiseaux sauvages dans la transmission et la dispersion des virus de l'influenza aviaire : apport de l'éco-épidémiologie dans les écosystèmes afro-tropicaux. Cah. Agric. 25: 54001. 